\title{
Comparative Intensity and Density Mapping of NGC 40
}

\author{
Trina L. Ray ${ }^{1}$ and C. T. Daub ${ }^{1}$
}

${ }^{1}$ Mount Laguna Observatory, San Diego State University

Two dimensional spectro-imaging of the planetary nebula NGC 40 was done to create spatially resolved intensity and electron density images. The CCD images, taken in $\mathrm{H} \beta$, [S II] $\lambda 6717$, and [S II] $\lambda \lambda 6717,6731$, were calibrated, then correlated using previously published spectroscopic and photometric data of NGC 40 as a flux standard.

There is a clear correspondence between the intensity and density images shown below. The bright lobes of NGC 40 are not associated with the highest densities, but rather are aligned along moderate density troughs. The highest densities we recorded are on the extremities of the nebula, beyond the bright lobes, but there are some localized high density regions toward the nebular interior ("clumps") associated with localized increases in intensity.

Dome basic modeling has been done in an attempt to identify a 3-D structure that reproduces the essential features of the observed 2-D intensity and density maps. The most successful model has inner and outer shells with high densities and low filling factors; and an emission-dominating middle shell with moderate density and high filling factor. The 'polar regions' of the shells are absent and the line-of-sight view is virtually equator-on.

A straightforward interpretation of the model suggests a stellar wind that sweeps the central region of the nebula clear and impinges upon matter previously ejected, causing the dense and inhomogeneous inner shell, perhaps via condensations with neutral cores. The middle shell is a homogeneous region of moderate density that dominates the nebular emission and whose outer boundary is marked by an ionization front. The associated shock front compresses the material in the outer, primarily neutral, shell producing the higher density there.
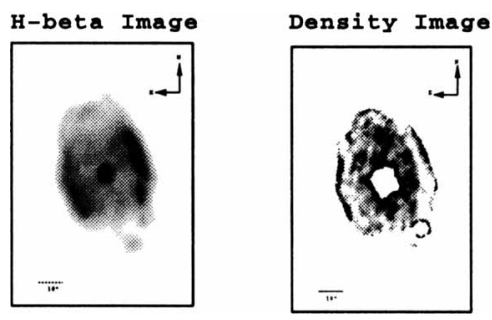

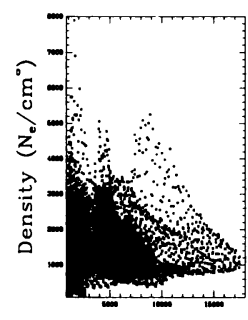

$\mathrm{H} \beta$ Intensity

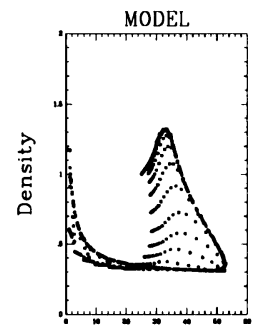

Intensity 